\title{
Quasilinear description of radiofrequency-induced radial diffusion
}

\section{Brambilla}

\author{
Max-Planck-Institut für Plasmaphysik-Euratom Association
}

Boltzmannstrasse 2, D-85748, Garching, Germany

\begin{abstract}
We derive the bounce-averaged quasilinear operator in axisymmetric toroidal geometry using the standard quasilinear formalism. When specialized to resonant ion cyclotron harmonic interactions, this operator predicts the same radio-frequency-induced radial diffusion as the orbit-averaged operator (L.-G. Eriksson and P. Helander, Phys. Plasmas 1 (1994) 308) obtained using action-angle variables. By assuming the wave field known as a superposition of toroidal and poloidal Fourier modes, the quasilinear diffusion coefficients are written in a form which can be directly evaluated using the output of a spectral full-wave solver of Maxwell equations in toroidal plasmas.
\end{abstract}

1 - Introduction. The fact that in toroidal geometry resonant cyclotron interactions inevitably cause radial diffusion of the heated ions is known since a long time [1]. As pointed out by Ryopulos et al. [2] and Hellsten [3], this is a consequence of the fact that the variation of the magnetic moment $\mu$ suffered by an ion transiting through a cyclotron resonance also causes a variation of its toroidal momentum $P_{\varphi}$. This, in turn, causes the mean radius of the drift orbit to shift radially outwards or inwards, depending on the sign of the toroidal wavenumber of the hf wave. The effect has been demonstrated experimentally in the JET tokamak by launching a toroidally asymmetric spectrum of fast waves [4].

An elegant form of the quasilinear (QL) kinetic equation including a term describing rf-induced radial diffusion has been derived by Eriksson and Helander [5]. A Monte Carlo method for its solution has been developed [5-7], and has since been successfully used for the interpretation of high-power ion cyclotron (IC) heating experiments in JET [8-9]. A review of recent work along these lines can be found in [10].

The derivations of [1] and [5] are based on the action-angle variables formalism of Kaufman [11]. This approach has been called 'orbit-averaging', to distinguish it from conventional 'bounce-averaging' [12], which is thought to be valid only in the limit of thin banana orbits. In this note we show, however, that the standard bounce-averaging procedure predicts just the same radial diffusion as orbit-averaging, unless it is explicitly or tacitly assumed that all charged particles are tied to magnetic field lines. We argue, therefore, that the real difference between orbit and bounce averaging lies in the treatment of collisions, which in Montecarlo codes, but not in bounce-averaged Fokker-Planck solvers, can be evaluated at the true position of the ions, independently from the shape and thickness of their orbit.

Our derivation differs from previous ones also in an other minor technical aspect, which is, however, of practical relevance. Most derivations of the QL operator assume that the wave field in the vicinity of IC resonances can be represented in Eikonal form. Here the wave electric field is supposed instead to be given as a superposition of toroidal and poloidal Fourier modes. This is the representation used to solve Maxwell equations in tokamak plasmas with spectral full-wave codes, e.g. TORIC [13]. In this way we obtain the quasilinear diffusion coefficients in a form suitable to be coded using the output of the code which solves the wave equations.

The rest of this note is organized as follows. In the next section we sketch the derivation of the QL operator from Vlasov equation, with the formalism we have previously used to obtain the constitutive relation of axisymmetric toroidal plasmas [14]. In section 3 bounce averaging is performed and briefly discussed. In section 4 the general results of the previous sections are specialized to cyclotron harmonic resonant interactions of ions, and the equivalence between bounce and orbit averaging is demonstrated. The last section contains some additional comments and the conclusions. 
2 - The quasilinear operator. In the quasilinear approximation, the quasi-steady-state distribution function is assumed to evolve slowly (compared to the frequency of the waves) under the effects of the time-average of the nonlinear term of Vlasov equation and of collisions,

$$
\frac{d F_{\alpha}}{d t} \equiv \frac{\partial F_{\alpha}}{\partial t}+\left(\vec{v}_{D}+v_{\|} \vec{b}_{0}\right) \cdot \vec{\nabla} F_{\alpha}+\frac{Z e}{m} E_{0 \|} \vec{b}_{0} \cdot \frac{\partial F_{\alpha}}{\partial \vec{v}}=\mathrm{C}\left(F_{\alpha}\right)+\mathrm{Q}\left(F_{\alpha}\right)
$$

On the lh side $\vec{v}_{D}$ is the drift velocity, $\vec{b}_{0}=\vec{B}_{0} / B_{0}$ is a unit vector in the direction of the static magnetic field, and $E_{0 \|}$ is the ohmic field, which is important for the electrons but normally negligible for the ions. On the rh side, $\mathrm{C}\left(F_{\alpha}\right)$ is the Landau collision operator, and $\mathrm{Q}\left(F_{\alpha}\right)$ the quasilinear operator, formally

$$
Q\left(F_{\alpha}\right)=-\frac{Z_{\alpha} e}{2 m_{\alpha}} \operatorname{Re}\left\langle\frac{\partial}{\partial \vec{v}} \cdot\left[\left(\vec{E}+\frac{\vec{v}}{c} \times \vec{B}\right)^{*} f_{\alpha}\right]\right\rangle_{t, \phi_{v}}
$$

where $f_{\alpha}$ is the solution of the linearized Vlasov equation driven by the h.f. fields, and the average is to be performed over time and over the gyration angle $\phi_{v}$.

The velocity derivative in eqn (2) is a divergence in velocity space. In axisymmetric configurations it must be written in terms of the (adiabatic) constants of the motion $\epsilon_{v}=v^{2} / 2$ and $\mu_{v}=v_{\perp}^{2} / 2 B_{0}$, and the third adiabatic invariant $\bar{\Psi}_{p}[15]$

$$
\bar{\Psi}_{p}=\bar{\Psi}_{g}-\frac{m c}{Z e}\left[R v_{\|}\left(\vec{b}_{0} \cdot \vec{u}_{\varphi}\right)-\omega_{B} \oint R\left(\vec{b}_{0} \cdot \vec{u}_{\varphi}\right) d s\right]
$$

where $\bar{\Psi}_{g}$ is the poloidal flux at the guiding center, and $\omega_{B}$ the transit or bouncing frequency. The integral on the r.h. side vanishes in the case of banana orbits, while in the case of passing particles it nearly cancels the first term. Referred to the poloidal flux at the radial position $\psi$ of the particle, therefore, we will approximate

$$
\bar{\Psi}_{p} \simeq \Psi_{p}(\psi)+\frac{\left(\vec{b} \times \vec{\nabla} \Psi_{p}\right) \cdot \vec{v}_{\perp}}{\Omega_{g}}-H_{\mathrm{tp}} B_{0} R \frac{v_{\|}}{\Omega_{c \alpha}} \cos \Theta
$$

where $\tan \Theta=B_{\mathrm{pol}} / B_{\mathrm{tor}}$, and the Heavyside function $H_{\mathrm{tp}}$ is unity for trapped and zero for passing particles (this amounts to neglect the radial excursions of passing particles from the average magnetic surface). In the collisionless limit, sufficient to evaluate the perturbed distribution function $f_{\alpha}, F_{\alpha}$ will be a function of $\epsilon_{v}, \mu_{v}$, and $\bar{\Psi}_{p}$. Taylor expanding $F_{\alpha}$ around the position of individual particles, we then obtain

$$
F_{\alpha}\left(\epsilon_{v}, \mu_{v}, \bar{\Psi}_{g}\right) \simeq F_{\alpha}\left(\epsilon_{v}, \mu_{v}, \psi\right)+\frac{\vec{K}_{B} \cdot \vec{v}}{\Omega_{g}} \frac{\partial F_{\alpha}}{\partial \psi}
$$

where $\vec{K}_{B}$ is a vector with the dimension of an inverse length, with components

$$
K_{B \psi}=0 \quad K_{B \eta}=\frac{N_{\vartheta}}{J_{p}} \quad K_{B \zeta}=-H_{\mathrm{tp}} \frac{B_{0} R}{\mathcal{F}_{P}} \cos \Theta
$$

(here $R J_{p}$ is the Jacobian, $N_{\vartheta}^{2}=g_{\vartheta \vartheta}$, and $\mathcal{F}(\psi)=d \Psi_{p} / d \psi$, cfr. [14]). The term of $\vec{K}_{B} \cdot \vec{v}$ proportional to $K_{B \eta}$ is the familiar diamagnetic contribution, which exists also in the straight limit. The other term is a 'neoclassical' correction due to toroidicity, which is predominatly (entirely in our approximation) contributed by trapped particles. The formal solution of the linearized Vlasov equation can then be written

$$
\begin{aligned}
f_{\alpha}(\vec{r}, \vec{v}, t)=-e^{-i \omega t} \frac{e Z_{\alpha}}{m_{\alpha}} \int_{-\infty}^{t} d t^{\prime} e^{i \omega\left(t-t^{\prime}\right)}\left[\left(\vec{E}\left(\vec{r}^{\prime}\right)+\frac{\vec{v}^{\prime}}{c} \times \vec{B}\left(\vec{r}^{\prime}\right)\right) .\right. \\
\left.\cdot\left(\vec{v}^{\prime} \frac{\partial F_{\alpha}}{\partial \epsilon_{v}}+\frac{\vec{v}_{\perp}^{\prime}}{B_{0}^{\prime}} \frac{\partial F_{\alpha}}{\partial \mu_{v}}+\frac{\vec{K}_{B}^{\prime}}{\Omega_{\alpha}^{\prime}} \frac{\partial F_{\alpha}}{\partial \psi}\right)\right]
\end{aligned}
$$


Inserting $f_{\alpha}$ into $(2)$ and using the rule of chain derivatives, we can write $Q\left(F_{\alpha}\right)$ as a diffusion operator

$$
\begin{aligned}
Q\left(F_{\alpha}\right)=\frac{v_{\|}}{B_{0}}\left\{\frac{\partial}{\partial \epsilon_{v}}\left[\frac{B_{0}}{v_{\|}}\left(\mathcal{D}_{\epsilon_{v} \epsilon_{v}} \frac{\partial F_{\alpha}}{\partial \epsilon_{v}}+\mathcal{D}_{\epsilon_{v} \mu_{v}} \frac{\partial F_{\alpha}}{\partial \mu_{v}}+\mathcal{D}_{\epsilon_{v} \psi} \frac{\partial F_{\alpha}}{\partial \psi}\right)\right]\right. \\
+\frac{\partial}{\partial \mu_{v}}\left[\frac{B_{0}}{v_{\|}}\left(\mathcal{D}_{\mu_{v} \epsilon_{v}} \frac{\partial F_{\alpha}}{\partial \epsilon_{v}}+\mathcal{D}_{\mu_{v} \mu_{v}} \frac{\partial F_{\alpha}}{\partial \mu_{v}}+\mathcal{D}_{\mu_{v} \psi} \frac{\partial F_{\alpha}}{\partial \psi}\right)\right] \\
\left.+\frac{1}{v_{\|}} \frac{\partial}{\partial \psi}\left[\frac{B_{0}}{v_{\|}}\left(\mathcal{D}_{\psi \epsilon_{v}} \frac{\partial F_{\alpha}}{\partial \epsilon_{v}}+\mathcal{D}_{\psi \mu_{v}} \frac{\partial F_{\alpha}}{\partial \mu_{v}}+\mathcal{D}_{\psi \psi} \frac{\partial F_{\alpha}}{\partial \psi}\right)\right]\right\}
\end{aligned}
$$

Here the quasilinear diffusion coefficients (QLDC) of the terms involving only velocity space dervatives are

$$
\begin{aligned}
& \mathcal{D}_{\epsilon_{v} \epsilon_{v}}=\frac{e^{2} Z_{\alpha}^{2}}{2 m_{\alpha}^{2}} \int_{0}^{2 \pi} \frac{d \phi_{v}}{2 \pi} \operatorname{Re}\left[\vec{v} \cdot \vec{E}^{*}(\vec{r}) \int_{-\infty}^{t} d t^{\prime} e^{i \omega\left(t-t^{\prime}\right)} \vec{E}\left(\vec{r}^{\prime}\right) \cdot \vec{v}^{\prime}\right] \\
& \mathcal{D}_{\epsilon_{v} \mu_{v}}=\frac{e^{2} Z_{\alpha}^{2}}{2 m_{\alpha}^{2}} \int_{0}^{2 \pi} \frac{d \phi_{v}}{2 \pi} \operatorname{Re}\left[\vec{v} \cdot \vec{E}^{*}(\vec{r}) \int_{-\infty}^{t} d t^{\prime} e^{i \omega\left(t-t^{\prime}\right)}\left(\vec{E}\left(\vec{r}^{\prime}\right)+\frac{v_{\|}^{\prime}}{c} \vec{b}_{0}^{\prime} \times \vec{B}\left(\vec{r}^{\prime}\right)\right) \cdot \frac{\vec{v}_{\perp}^{\prime}}{B_{0}^{\prime}}\right] \\
& \mathcal{D}_{\mu_{v} \epsilon_{v}}=\frac{e^{2} Z_{\alpha}^{2}}{2 m_{\alpha}^{2}} \int_{0}^{2 \pi} \frac{d \phi_{v}}{2 \pi} \operatorname{Re}\left[\frac{\vec{v}_{\perp}}{B_{0}} \cdot\left(\vec{E}(\vec{r})+\frac{v_{\|}}{c} \vec{b}_{0} \times \vec{B}(\vec{r})\right)^{*} \int_{-\infty}^{t} d t^{\prime} e^{i \omega\left(t-t^{\prime}\right)} \vec{E}\left(\vec{r}^{\prime}\right) \cdot \vec{v}^{\prime}\right] \\
& \mathcal{D}_{\mu_{v} \mu_{v}}=\frac{e^{2} Z_{\alpha}^{2}}{2 m_{\alpha}^{2}} \int_{0}^{2 \pi} \frac{d \phi_{v}}{2 \pi} \operatorname{Re}\left[\frac{\vec{v}_{\perp}}{B_{0}} \cdot\left(\vec{E}(\vec{r})+\frac{v_{\|}}{c} \vec{b}_{0} \times \vec{B}(\vec{r})\right)^{*} t^{\prime} e^{i \omega\left(t-t^{\prime}\right)}\left(\vec{E}\left(\vec{r}^{\prime}\right)+\frac{v_{\|}^{\prime}}{c} \vec{b}_{0}^{\prime} \times \vec{B}\left(\vec{r}^{\prime}\right)\right) \cdot \frac{\vec{v}_{\perp}^{\prime}}{B_{0}^{\prime}}\right]
\end{aligned}
$$

while the QLDC involving also radial derivatives are

$$
\begin{aligned}
& \mathcal{D}_{\epsilon_{v} \psi}=\frac{e^{2} Z_{\alpha}^{2}}{2 m_{\alpha}^{2}} \int_{0}^{2 \pi} \frac{d \phi_{v}}{2 \pi} \operatorname{Re}\left[\vec{v} \cdot \vec{E}^{*}(\vec{r}) \int_{-\infty}^{t} d t^{\prime} e^{i \omega\left(t-t^{\prime}\right)}\left(\vec{E}\left(\vec{r}^{\prime}\right)+\frac{\vec{v}^{\prime}}{c} \times \vec{B}\left(\vec{r}^{\prime}\right)\right) \cdot \frac{\vec{K}_{B}^{\prime}}{\Omega_{g}^{\prime}}\right] \\
& \mathcal{D}_{\mu_{v} \psi}=\frac{e^{2} Z_{\alpha}^{2}}{2 m_{\alpha}^{2}} \int_{0}^{2 \pi} \frac{d \phi_{v}}{2 \pi} \operatorname{Re}\left[\frac{\vec{v}_{\perp}}{B_{0}} \cdot\left(\vec{E}(\vec{r})+\frac{v_{\|}}{c} \vec{b}_{0} \times \vec{B}(\vec{r})\right)^{*}\right. \\
& \left.\times \int_{-\infty}^{t} d t^{\prime} e^{i \omega\left(t-t^{\prime}\right)}\left(\vec{E}\left(\vec{r}^{\prime}\right)+\frac{\vec{v}^{\prime}}{c} \times \vec{B}\left(\vec{r}^{\prime}\right)\right) \cdot \frac{\vec{K}_{B}^{\prime}}{\Omega_{g}^{\prime}}\right] \\
& \mathcal{D}_{\psi \epsilon_{v}}=-\frac{e^{2} Z_{\alpha}^{2}}{2 m_{\alpha}^{2}} \int_{0}^{2 \pi} \frac{d \phi_{v}}{2 \pi} \operatorname{Re}\left[\frac{\vec{K}_{B}}{\Omega_{g}} \cdot\left(\vec{E}(\vec{r})+\frac{\vec{v}_{\perp}}{c} \times \vec{B}(\vec{r})\right)^{*} \int_{-\infty}^{t} d t^{\prime} e^{i \omega\left(t-t^{\prime}\right)} \vec{E}\left(\vec{r}^{\prime}\right) \cdot \vec{v}^{\prime}\right] \\
& \mathcal{D}_{\psi \mu_{v}}=-\frac{e^{2} Z_{\alpha}^{2}}{2 m_{\alpha}^{2}} \int_{0}^{2 \pi} \frac{d \phi_{v}}{2 \pi} \operatorname{Re}\left[\frac{\vec{K}_{B}}{\Omega_{g}} \cdot\left(\vec{E}(\vec{r})+\frac{\vec{v}_{\perp}}{c} \times \vec{B}(\vec{r})\right)^{*}\right. \\
& \left.\times \int_{-\infty}^{t} d t^{\prime} e^{i \omega\left(t-t^{\prime}\right)}\left(\vec{E}\left(\vec{r}^{\prime}\right)+\frac{v_{\|}^{\prime}}{c} \vec{b}_{0}^{\prime} \times \vec{B}\left(\vec{r}^{\prime}\right)\right) \cdot \frac{\vec{v}_{\perp}^{\prime}}{B_{0}^{\prime}}\right] \\
& \mathcal{D}_{\psi \psi}=-\frac{e^{2} Z_{\alpha}^{2}}{2 m_{\alpha}^{2}} \int_{0}^{2 \pi} \frac{d \phi_{v}}{2 \pi} \operatorname{Re}\left[\frac{\vec{K}_{B}}{\Omega_{g}} \cdot\left(\vec{E}(\vec{r})+\frac{\vec{v}_{\perp}}{c} \times \vec{B}(\vec{r})\right)^{*}\right. \\
& \left.\times \int_{-\infty}^{t} d t^{\prime} e^{i \omega\left(t-t^{\prime}\right)}\left(\vec{E}\left(\vec{r}^{\prime}\right)+\frac{\vec{v}_{\perp}^{\prime}}{c} \times \vec{B}\left(\vec{r}^{\prime}\right)\right) \cdot \frac{\vec{K}_{B}^{\prime}}{\Omega_{g}^{\prime}}\right]
\end{aligned}
$$

The presence of radial derivatives in $Q\left(F_{\alpha}\right)$ illustrates the fact that in nonuniform configurations diffusion in velocity space and in real space are not independent. According to the quasilinear picture, resonant wave-particle interactions act somewhat like collisions, by modifying in small random steps the value of the adiabatic constants of the unperturbed motion. Since in toroidal geometry these constants depend 
on the radial coordinate, radial diffusion necessarily follows. The elementary step of the classical term is the Larmor radius, resulting in a diffusion which is negligible in practice. Neoclassical radial diffusion induced by resonant interactions with $\mathrm{rf}$ waves, by contrast, can be quite important for energetic trapped particles, for which the elementary step is a fraction of the radial width of the banana orbit. In the following, therefore, we will retain only the neoclassical term.

Introducing the spectral representation of the fields [14]

$$
\left(\begin{array}{l}
\vec{E}(\vec{r}, t) \\
\vec{B}(\vec{r}, t)
\end{array}\right)=\sum_{m, n} \int d \kappa\left(\begin{array}{c}
\overrightarrow{\mathcal{E}}^{m n}(\kappa) \\
\overrightarrow{\mathcal{B}}^{m n}(\kappa)
\end{array}\right) e^{i(\kappa \psi+m \vartheta+n \varphi-\omega t)}
$$

we can rewrite (7) as

$$
Q\left(F_{\alpha}\right)=\frac{v_{\|}}{B_{0}}\left[\frac{\partial}{\partial \epsilon_{v}}\left(\frac{B_{0}}{v_{\|}} \Gamma^{\epsilon_{v}}\right)+\frac{\partial}{\partial \mu_{v}}\left(\frac{B_{0}}{v_{\|}} \Gamma^{\mu_{v}}\right)+\frac{1}{v_{\|}} \frac{\partial}{\partial \psi}\left(\frac{B_{0}}{v_{\|}} \Gamma^{\psi}\right)\right]
$$

where

$$
\begin{aligned}
\Gamma^{\epsilon_{v}} & =\mathcal{D}_{\epsilon_{v} \epsilon_{v}} \frac{\partial F_{\alpha}}{\partial \epsilon_{v}}+\mathcal{D}_{\epsilon_{v} \mu_{v}} \frac{\partial F_{\alpha}}{\partial \mu_{v}}+\mathcal{D}_{\epsilon_{v} \psi} \frac{\partial F_{\alpha}}{\partial \psi} \\
\Gamma^{\mu_{v}} & =\mathcal{D}_{\mu_{v} \epsilon_{v}} \frac{\partial F_{\alpha}}{\partial \epsilon_{v}}+\mathcal{D}_{\mu_{v} \mu_{v}} \frac{\partial F_{\alpha}}{\partial \mu_{v}}+\mathcal{D}_{\mu_{v} \psi} \frac{\partial F_{\alpha}}{\partial \psi} \\
\Gamma^{\psi} & =\mathcal{D}_{\psi \epsilon_{v}} \frac{\partial F_{\alpha}}{\partial \epsilon_{v}}+\mathcal{D}_{\psi \mu_{v}} \frac{\partial F_{\alpha}}{\partial \mu_{v}}+\mathcal{D}_{\psi \psi} \frac{\partial F_{\alpha}}{\partial \psi}
\end{aligned}
$$

Some lengthy but straightforward algebra, best performed in rotating components, gives

$$
\begin{aligned}
\mathcal{D}_{\gamma \delta}=\frac{Z_{\alpha}^{2} e^{2}}{2 m_{\alpha}^{2}} \operatorname{Re}\left[\int d \kappa_{1} \int d \kappa_{2} \sum_{m_{1}, m_{2}} \sum_{n_{1}, n_{2}} e^{i\left[\left(\kappa_{2}-\kappa_{1}\right) \psi_{g}+\left(m_{2}-m_{1}\right) \vartheta_{g}+\left(n_{2}-n_{1}\right) \varphi_{g}\right]}\right. & \\
& \left.\times \sum_{p} \Delta_{\gamma}^{*}\left(p \mid \vec{k}_{1}\right) \int_{-\infty}^{t} \mathcal{G}_{p}^{\prime}\left(\vec{k}_{2}\right) \Delta_{\delta}^{\prime}\left(p \mid \vec{k}_{2}\right)\right]
\end{aligned}
$$

with

$$
\begin{aligned}
& \Delta_{\epsilon_{v}}(p \mid \vec{k})=\frac{v_{\perp}}{\sqrt{2}}\left[J_{p-1}(\nu w) \mathcal{E}_{+}(\vec{k}) e^{-i \delta}+J_{p+1}(\nu w) \mathcal{E}_{-}(\vec{k}) e^{+i \delta}\right]+v_{\|} J_{p}(\nu w) \mathcal{E}_{\zeta}(\vec{k}) \\
& =v_{t h \alpha}\left[\frac{p}{\nu} J_{p}(\nu w) \frac{\overrightarrow{\mathcal{E}}(\vec{k}) \cdot \vec{k}_{\perp}}{k_{\perp}}-i w J_{p}^{\prime}(\nu w) \frac{\left[\vec{k}_{\perp} \times \overrightarrow{\mathcal{E}}(\vec{k})\right] \cdot \vec{b}_{0}}{k_{\perp}}+u J_{p}(\nu w)\left(\overrightarrow{\mathcal{E}}(\vec{k}) \cdot \vec{b}_{0}\right)\right] \\
& \Delta_{\mu_{v}}(p \mid \vec{k})=\frac{v_{\perp}}{\sqrt{2} B_{0}}\left[J_{p-1}(\nu w)\left(\mathcal{E}_{+}(\vec{k})+i \frac{v_{\|}}{c} \mathcal{B}_{+}(\vec{k})\right) e^{-i \delta}\right. \\
& \left.+J_{p+1}(\nu w)\left(\mathcal{E}_{-}(\vec{k})-i \frac{v_{\|}}{c} \mathcal{B}_{-}(\vec{k})\right) e^{+i \delta}\right] \\
& =\frac{v_{t h \alpha}}{B_{0}}\left[\frac{p}{\nu} J_{p}(\nu w)\left(\frac{\overrightarrow{\mathcal{E}}(\vec{k}) \cdot \vec{k}_{\perp}}{k_{\perp}}-\frac{v_{\|}}{c} \frac{\left[\vec{k}_{\perp} \times \overrightarrow{\mathcal{B}}(\vec{k})\right] \cdot \vec{b}_{0}}{k_{\perp}}\right)\right. \\
& \left.-i w J_{p}^{\prime}(\nu w)\left(\frac{\left[\vec{k}_{\perp} \times \overrightarrow{\mathcal{E}}(\vec{k})\right] \cdot \vec{b}_{0}}{k_{\perp}}-\frac{v_{\|}}{c} \frac{\overrightarrow{\mathcal{B}}(\vec{k}) \cdot \vec{k}_{\perp}}{k_{\perp}}\right)\right] \\
& \Delta_{\psi}(p \mid \vec{k})=K_{B \zeta}\left[J_{p}(\nu w) \mathcal{E}_{\zeta}(\vec{k})-i \frac{v_{\perp}}{\sqrt{2} c}\left(J_{p-1}(\nu w) \mathcal{B}_{+}(\vec{k}) e^{-i \delta}-J_{p+1}(\nu w) \mathcal{B}_{-}(\vec{k}) e^{i \delta}\right)\right] \\
& =K_{B \zeta}\left[J_{p}(\nu w) \overrightarrow{\mathcal{E}}(\vec{k}) \cdot \vec{b}_{0}+\frac{v_{\perp}}{c}\left(\frac{p}{\nu w} J_{p}(\nu w) \frac{\left(\vec{k}_{\perp} \times \overrightarrow{\mathcal{B}}(\vec{k})\right) \cdot \vec{b}_{0}}{k_{\perp}}-i J_{p}^{\prime}(\nu w) \frac{\vec{k}_{\perp} \cdot \overrightarrow{\mathcal{B}}(\vec{k})}{k_{\perp}}\right)\right]
\end{aligned}
$$

where $w=v_{\perp} / v_{t h \alpha}, \nu=k_{\perp} v_{t h \alpha} / \Omega_{c \alpha}$, and $\delta=\tan ^{-1}\left(k_{\eta} / k_{\psi}\right)$. It can be useful to note that each $\Delta_{K}$ has the dimension of the rate of time variation of the corresponding adiabatic constant of the motion. 
3 - The bounce-averaged quasilinear equation. Although $Q(F)$ depends explicitly on all three coordinates $\psi, \vartheta$, and $\varphi$, the time variation of $F_{\alpha}$ is expected to occur on a much slower time scale than the bounce motion of most particles. This allows to 'bounce average' eqn (11), thereby eliminating the $\vartheta$ and $\varphi$ dependencies. To perform the averaging, let us write the convective term in r.h. side in symmetric flux coordinates

$$
\frac{\partial F_{\alpha}}{\partial t}+\vec{v}_{D} \cdot \vec{\nabla} F_{\alpha}+\frac{\mathcal{F}_{P}}{R J_{p}} \frac{v_{\|}}{B_{0}}\left(\frac{\partial F_{\alpha}}{\partial \vartheta}+q \frac{\partial F_{\alpha}}{\partial \varphi}\right)=S\left(F_{\alpha}\right)+Q\left(F_{\alpha}\right)
$$

Because of axisymmetry, averaging over the toroidal angle is trivial: it eliminates the $\varphi$ (toroidal angle) derivative from the 1.h. side, and reduces the expressions listed above for $\mathcal{D}_{\alpha \beta}$ to sums of independent contributions from each toroidal Fourier component of the wave field. Symbolically,

$$
\left\langle\sum_{n, n^{\prime}} \mathcal{D}_{\gamma \delta}\left(n, n^{\prime}\right) e^{i\left(n^{\prime}-n\right) \varphi}\right\rangle_{\varphi} \rightarrow \sum_{n, n^{\prime}} \mathcal{D}_{\gamma \delta}\left(n, n^{\prime}\right) \delta\left(n^{\prime}-n\right) \rightarrow \sum_{n} \mathcal{D}_{\gamma \delta}(n)
$$

In the following the $\varphi$ averaging step will not be explicitly indicated. To average eqn (15) over the poloidal angle, on the other hand, we first multiply both sides by $\left(R J_{p} / \mathcal{F}_{P}\right)\left(B_{0} / v_{\|}\right)=\left(N_{\vartheta} / B_{\mathrm{pol}}\right)\left(B_{0} / v_{\|}\right)$. Integrating over $\vartheta$ then eliminates the convective term due to the bounce motion:

$$
\frac{\partial F_{\alpha}}{\partial t}+\left\langle v_{D \psi} \frac{\partial F_{\alpha}}{\partial \psi}\right\rangle=S_{\text {avg }}\left(F_{\alpha}\right)+Q_{\text {avg }}\left(F_{\alpha}\right)
$$

where

$$
Q_{\mathrm{avg}}\left(F_{\alpha}\right)=\frac{1}{\left\langle\lambda_{B}\right\rangle}\left[\frac{\partial \bar{\Gamma}^{\epsilon_{v}}}{\partial \epsilon_{v}}+\frac{\partial \bar{\Gamma}^{\mu_{v}}}{\partial \mu_{v}}+\frac{1}{R J_{p}} \frac{\partial}{\partial \psi}\left(R N_{\vartheta} \bar{\Gamma}^{\psi}\right)\right]
$$

where

$$
\begin{aligned}
\bar{\Gamma}^{\epsilon_{v}} & =\overline{\mathcal{D}}_{\epsilon_{v} \epsilon_{v}} \frac{\partial F_{\alpha}}{\partial \epsilon_{v}}+\overline{\mathcal{D}}_{\epsilon_{v} \mu_{v}} \frac{\partial F_{\alpha}}{\partial \mu_{v}}+\overline{\mathcal{D}}_{\epsilon_{v} \psi} \frac{\partial F_{\alpha}}{\partial \psi} \\
\bar{\Gamma}^{\mu_{v}} & =\overline{\mathcal{D}}_{\mu_{v} \epsilon_{v}} \frac{\partial F_{\alpha}}{\partial \epsilon_{v}}+\overline{\mathcal{D}}_{\mu_{v} \mu_{v}} \frac{\partial F_{\alpha}}{\partial \mu_{v}}+\overline{\mathcal{D}}_{\mu_{v} \psi} \frac{\partial F_{\alpha}}{\partial \psi} \\
\bar{\Gamma}^{\psi} & =\frac{J_{p}}{N_{\vartheta}}\left(\overline{\mathcal{D}}_{\psi \epsilon_{v}} \frac{\partial F_{\alpha}}{\partial \epsilon_{v}}+\overline{\mathcal{D}}_{\psi \mu_{v}} \frac{\partial F_{\alpha}}{\partial \mu_{v}}+\overline{\mathcal{D}}_{\psi \psi} \frac{\partial F_{\alpha}}{\partial \psi}\right)
\end{aligned}
$$

with

$$
\overline{\mathcal{D}}_{\gamma \delta}=\oint \frac{R J_{p}}{\mathcal{F}_{P}} \frac{B_{0}}{v_{\|}} \mathcal{D}_{\gamma \delta} d \vartheta=q \oint \frac{R h_{s}}{v_{\|}} \mathcal{D}_{\gamma \delta} d \vartheta
$$

and

$$
\left\langle v_{D \psi} \frac{\partial F}{\partial \psi}\right\rangle=\frac{1}{\left\langle\lambda_{B}\right\rangle} \oint \frac{R N_{\vartheta}}{v_{\|}} v_{D \psi} \frac{\partial F}{\partial \psi} d \vartheta
$$

In these equations

$$
\left\langle\lambda_{B}\right\rangle=\oint \frac{R J_{p}}{\mathcal{F}_{P}} \frac{B_{0}}{v_{\|}} d \vartheta=q \oint \frac{R h_{s}}{v_{\|}} d \vartheta
$$

In eqns (20) and (22) the second expression, with $h_{s}^{2}=1+N_{\vartheta}^{2} / q^{2} R^{2}$, holds only in flux coordinates, and with $h_{s} \simeq 1$ is an approximation valid to first order in the inverse aspect ratio in any coordinate system. Recalling that the line element along a magnetic field line is $d \ell_{s}=R h_{s} d \vartheta$, it is seen that the surface average is actually a time average: the integrand is weighted with the time spent by the particles in each element $d \ell_{s}$ of their trajectory. Because of the approximate form used for $\bar{\Psi}_{p}$, the QLDC $\overline{\mathcal{D}}_{\psi \beta}$ weighted with $\lambda_{B}$ have an integrable singularity at the boundary between the trapping and passing domains. 
Strictly speaking, the surface-averaged quasilinear operator (18) is no more in the form of a divergence in velocity space. Nevertheless, if rf-induced radial diffusion is neglected (i.e. if particles are assumed tied to magnetic field lines), it preserves the density

$$
\bar{n}(\psi)=\frac{2 \pi}{\bar{V}(\psi)} \int_{0}^{2 \pi} R J_{p} d \vartheta \iint \frac{B_{0}}{v_{\|}} F_{\alpha}\left(\epsilon_{v}, \mu_{v}\right) d \epsilon_{v} d \mu_{v}
$$

where $\bar{V}(\psi)=2 \pi \oint R J_{p} d \vartheta$ is the specific volume of the magnetic surface $\psi$. Indeed, interchanging the order of integrations taking into account that, by definition, $Q_{\mathrm{avg}}\left(F_{\alpha}\right)$ does not depend on $\vartheta$,

$$
\begin{aligned}
\frac{\partial \bar{n}}{\partial t} & =\frac{2 \pi}{\bar{V}(\psi)} \int_{0}^{2 \pi} R J_{p} d \vartheta \iint \frac{B_{0}}{v_{\|}} Q_{\mathrm{avg}}\left(F_{\alpha}\right) d \epsilon_{v} d \mu_{v} \\
& =\frac{2 \pi}{\bar{V}(\psi)} \iint\left\langle\lambda_{B}\right\rangle Q_{\mathrm{avg}}\left(F_{\alpha}\right) d \epsilon_{v} d \mu_{v}=0
\end{aligned}
$$

In other words, $\left\langle\lambda_{B}\right\rangle d \epsilon_{v} d \mu_{v}$ can be be regarded as the 'surface averaged' volume element in velocity space. In the same approximation, the quasilinear rate of change of the kinetic energy per unit volume of species $\alpha$, averaged over the magnetic surface, is

$$
\begin{aligned}
\frac{d \bar{K}_{\alpha}}{d t} & =\frac{2 \pi}{\bar{V}(\psi)} \int_{0}^{2 \pi} R J_{p} d \vartheta \int \epsilon_{v} d \epsilon_{v} \int \frac{B_{0}}{v_{\|}} d \mu_{v} \hat{Q}_{\mathrm{avg}}\left(F_{\alpha}\right) \\
& =-\frac{2 \pi}{\bar{V}(\psi)} \iint\left(\overline{\mathcal{D}}_{\epsilon_{v} \epsilon_{v}} \frac{\partial F_{\alpha}}{\partial \epsilon_{v}}+\overline{\mathcal{D}}_{\epsilon_{v} \mu_{v}} \frac{\partial F_{\alpha}}{\partial \mu_{v}}\right) d \epsilon_{v} d \mu_{v}
\end{aligned}
$$

Using the expressions for $\overline{\mathcal{D}}_{\alpha \beta}$ obtained above, and rearranging the order of the integrations, we can write more explicitly

$$
\begin{aligned}
\frac{d \bar{K}_{\alpha}}{d t}=- & \frac{\pi \omega}{2 \bar{V}(\psi)} \frac{\omega_{p \alpha}^{2}}{\omega^{2}} \int_{0}^{2 \pi} R J_{p} d \vartheta \frac{B_{0}}{v_{\|}} \operatorname{Im}\left\{\int d \kappa_{1} \int d \kappa_{2}\right. \\
& \times \sum_{m_{1}, m_{2}} \sum_{n} e^{i\left[\left(\kappa_{2}-\kappa_{1}\right) \psi+\left(m_{2}-m_{1}\right) \vartheta\right]} \int d \epsilon_{v} \int d \mu_{v} \sum_{p} \Delta_{\epsilon_{v}}^{*}\left(p \mid \vec{k}_{1}\right) \\
& \left.\times(-i \omega) \int_{-\infty}^{t} d t^{\prime} \mathcal{G}_{p}^{\prime}\left(\vec{k}_{2}\right)\left[\Delta_{\epsilon_{v}}^{\prime}\left(p \mid \vec{k}_{2}\right) \frac{\partial F_{\alpha}}{\partial \epsilon_{v}}+\Delta_{\mu_{v}}^{\prime}\left(p \mid \vec{k}_{2}\right) \frac{\partial F_{\alpha}}{\partial \mu_{v}}\right]\right\}
\end{aligned}
$$

It can be shown that this is identical with the wave absorption rate evaluated from the wave equations in the same approximation (particles tied to magnetic field lines).

If the rf induced radial diffusion is taken into account, the averaged density is no more constant; instead

$$
\frac{\partial \bar{n}}{\partial t}=\frac{2 \pi}{\bar{V}(\psi)} \int_{0}^{\infty} d \epsilon_{v} \int_{\epsilon_{v} / B_{0 M a x}}^{\epsilon_{v} / B_{0}} \frac{\partial}{\partial \psi}\left(\overline{\mathcal{D}}_{\psi \epsilon_{v}} \frac{\partial F_{\alpha}}{\partial \epsilon_{v}}+\overline{\mathcal{D}}_{\psi \mu_{v}} \frac{\partial F_{\alpha}}{\partial \mu_{v}}+\overline{\mathcal{D}}_{\psi \psi} \frac{\partial F_{\alpha}}{\partial \psi}\right) d \mu_{v}
$$

Inside a volume containing all paricle orbits (i.e., neglecting direct losses due to orbits interscting the wall), however, the total number of particles is conserved ${ }^{(1)}$. In writing (27) we have neglected the convective term on the lh side; this term has been evaluated by Chang [16] and by Chen et al. [17], who, however, have missed the neoclassical part in the quasilinear operator itself.

(1) To put the last term of (18) in the form appropriate to obtain this result, however, it has been necessary to bring the Jacobian $\left(R J_{p}\right)^{-1}$ outside the $\psi$ derivation. This seems acceptable to lowest order in the drift parameter. 
4-Quasilinear operator for IC harmonic interactions. For ions in the ion cyclotron (IC) frequency range we can in the first place approximate

$$
\overrightarrow{\mathcal{B}}(\vec{k})=\frac{c}{\omega} \vec{k} \times \overrightarrow{\mathcal{E}}(\vec{k})
$$

Note that this approximation has nothing to do with an Eikonal ansatz: it only amounts to neglect some logarithmic derivatives of the metrics compared to the wavevector, and must hold separately for each Fourier component in eqns (10). To obtain a more explicit result, and to facilitate the comparison with the QLDC obtained elsewhere, however, we next assume that IC resonances occur only with the Fast wave $(\mathrm{FW})$, and introduce for this wave the following generalized Eikonal Ansatz

$$
\begin{aligned}
\vec{E}(\vec{r}, t) & =\sum_{m n} \vec{E}_{0}^{m n}(\epsilon \psi) \exp i\left[\frac{S_{m, n}(\epsilon \psi, \epsilon \vartheta)}{\epsilon}+m \vartheta+n \varphi-\omega t\right] \\
& \simeq \sum_{m n} \vec{E}_{0}^{m n}(\epsilon \psi) e^{i\left[\kappa_{m, n} \psi+m \vartheta+n \varphi-\omega t\right]}
\end{aligned}
$$

where $\epsilon \ll 1$ is a small parameter denoting quantities which vary slowly compared to the wavector; it will be omitted in the following. In this case, for each Fourier mode $m, n$, the radial wavenumber $\kappa_{m, n}=\partial S_{m, n} / \partial \psi$ is uniquely determined (except for the sign) by the local dispersion relation of the FW. The ansatz (29), therefore, eliminates the integrations over $\kappa_{1}$ and $\kappa_{2}$ in the definition of $\overline{\mathcal{D}}_{\gamma \delta}$. We can now exploit the fact that by far the largest contribution to the QLDC comes from the immediate vicinity of the points where the wave phase seen by the particle (the phase of the 'propagator' $\mathcal{G}_{p}^{\prime}\left(\vec{k}_{2}\right)$ ) is stationary,

$$
\omega=p \Omega_{c \alpha}+\vec{k}_{2} \cdot\left(v_{\|} \vec{b}_{0}+\vec{v}_{D}\right)
$$

This condition defines two (four in case of toroidally trapped particles) resonant angles, which, however, can merge either because the resonances occurs near the equator of a magnetic surface (tangential resonances), or in the case of trapped particles which happen to reverse the parallel motion close to resonance. Disregarding for the moment these exceptional cases, the poloidal angle integration in eqn (6) can be performed by developing the phase to second order around resonances, to obtain

$$
\begin{aligned}
\overline{\mathcal{D}}_{\gamma \delta}^{p}=\frac{\pi Z_{\alpha}^{2} e^{2}}{2 m_{\alpha}^{2} \omega} \sum_{\text {res }}\left\{\frac{R J_{p}}{\mathcal{F}_{P}} \frac{B_{0}}{v_{\|}} \operatorname{Re}\right. & \sum_{m_{1}, m_{2}} \sum_{n} e^{i\left[\left(\kappa_{2}-\kappa_{1}\right) \psi+\left(m_{2}-m_{1}\right) \vartheta\right]} \\
& \left.\times \Delta_{\gamma}^{*}\left(p \mid \vec{k}_{1}\right) \mathcal{W}_{p}\left(\vec{k}_{2}, \psi, \vartheta\right) \Delta_{\delta}\left(p \mid \vec{k}_{2}\right)\right\}_{\vartheta=\vartheta_{\text {res }}}
\end{aligned}
$$

where

$$
\mathcal{W}_{p}\left(\vec{k}_{2}, \psi, \vartheta\right)=\frac{\omega}{\left|\partial\left[\vec{k}_{2} \cdot\left(v_{\|} \vec{b}_{0}+\vec{v}_{D}\right)+p \Omega_{c \alpha}\right] / \partial \vartheta\right|}
$$

can be called the 'resonance kernel' of the QL diffusion coefficients. In eqn (31), $\vec{k}_{1}$ and $\vec{k}_{2}$ have the same toroidal wavenumber $n$, and, according to the observation following the introduction of the ansatz (29), $\kappa_{1}=\kappa_{m_{1}, n}, \kappa_{2}=\kappa_{m_{2}, n}$. If we also neglect the effects of the small parallel component of the wave electric field, the three quantities $\Delta_{K}$ can now be expressed in terms of a single one,

$$
\Delta_{\epsilon_{v}}(p \mid \vec{k})=\mathrm{D}_{p}(\vec{k}, \psi) \quad \Delta_{\mu_{v}}(p \mid \vec{k})=\frac{1}{B_{0}} \mathrm{D}_{p}(\vec{k}, \psi) \quad \Delta_{\psi}(p \mid \vec{k})=-\frac{K_{B \zeta} k_{\zeta}}{\Omega_{c \alpha} \omega} \mathrm{D}_{p}(\vec{k}, \psi)
$$

(the $\psi$ dependency is reintroduced by the ansatz (29)), with

$$
\begin{aligned}
\mathrm{D}_{p}(\vec{k}, \psi) & =v_{t h \alpha}\left(\frac{p}{\nu} J_{p}(\nu w) \frac{\vec{E}_{0 \perp}^{m n}(\psi) \cdot \vec{k}_{\perp}}{k_{\perp}}-i w J_{p}^{\prime}(\nu w) \frac{\left[\vec{k}_{\perp} \times \vec{E}_{0}^{m n}(\psi)\right] \cdot \vec{b}}{k_{\perp}}\right) \\
& =\frac{v_{\perp}}{\sqrt{2}}\left(J_{p-1}(\nu w) E_{0+}^{m n}(\psi) e^{-i \delta}+J_{p+1}(\nu w) E_{0-}^{m n}(\psi) e^{+i \delta}\right)
\end{aligned}
$$


Finally, we note that the resonance kernel (32) has an integrable singularity in the exceptional cases mentioned above. How to improve its evaluation in the case of merging resonances so as to avoid this divergence is well-known [18-19], and we will omit this discussion here.

The QL operator obtained by substituting (31)-(34) into eqns (18)-(19) can alternativelt be written

$$
\begin{aligned}
Q_{\text {avg }}\left(F_{\alpha}\right)=\frac{1}{\left\langle\lambda_{B}\right\rangle} \sum_{\text {res }}\left(\frac{\partial}{\partial \epsilon_{v}}+\frac{1}{B_{0}} \frac{\partial}{\partial \mu_{v}}\right. & \left.-\frac{K_{B \zeta} k_{\zeta}}{\Omega_{c \alpha} \omega} \frac{1}{R J_{p}} \frac{\partial}{\partial \psi}\right)\left[\left|\mathrm{D}_{p}\right|^{2}\right. \\
& \left.\times\left(\frac{\partial F_{\alpha}}{\partial \epsilon_{v}}+\frac{1}{B_{0}} \frac{\partial F_{\alpha}}{\partial \mu_{v}}-\frac{K_{B \zeta} k_{\zeta}}{\Omega_{c \alpha} \omega} \frac{1}{R J_{p}} \frac{\partial F_{\alpha}}{\partial \psi}\right)\right]
\end{aligned}
$$

with the convention that the coefficients for the derivatives inside the round brackets, except for the metric factor $\left(R J_{P}\right)^{-1}$, have to be taken at resonance. In this form, it is easily seen to differ from the orbit-averaged QL operator of reference [5] only because it is written in different phase-space variables (and because of the approximate expression we have used for the third invariant). In particular, the coefficients of spatial diffusion are proportional to the parallel component $k_{\zeta}$ of the wavevector, and will cause inward or outward diffusion depending on its sign.

5 - Discussion and conclusions. The form (35) of the quasilinear operator (and even better the form proposed in [5]) is advantageous for the development of the Montecarlo technique for the solution of the QL equation [5-7]. This approach allows to take into account the effects of collisions at the true position of the ions, even when their orbits explore a substantial fraction of the plasma radius. It also facilitates the detailed discussion of the effects of resonant IC interactions on very energetic ions on large banana or non-standard orbits [10]. The completely equivalent expression (18), in the form of the divergence of a flux in phase space, on the other hand, has the advantage of being a straighforward generalization of the QL operator used in standard solvers of the surface averaged Fokker-Planck (FP) equation. In such codes the effects of collisions are less accurately evaluated at the average magnetic surface. In most situations, however, this should be a sufficient approximation for ions at intermediate energies, which due to the exponental decrease of the distribution functions with energy can neverteless be expected to build the vast majority of the suprathermal population. Extending the capability of a FP solver to deal with the radial dimension using the results of this note should be relatively straightforward, although demanding from the point of view of computing resources. In this context, we can add two comments.

First we note that each component of the rf-induced flux (19) in phase space has a term proportional to the radial gradient of the distribution function. In the few cases in which radial diffusion has been taken into account in a FP solver, only the diagonal term has been considered, omitting the mixed terms.

The second comment concerns the use of the output of a spectral solver of Maxwell equations in the plasma [13] to build the QLDC of eqns (31)-(34). In a full-wave code, the electric field is actually obtained in the form

$$
\vec{E}(\vec{r}, t)=\sum_{m n} \vec{E}^{m n}(\psi) e^{i(m \vartheta+n \varphi-\omega t)}
$$

without introducing the Eikonal ansatz (29) for the radial dependence of the coefficients $\vec{E}^{m n}(\psi)$. This simply means that $\vec{E}^{m n}(\psi)$ must replace $\vec{E}_{0}^{m n}(\psi) e^{i \kappa_{m n} \psi}$ in eqn (34), and eliminates the ambiguity in this equation due to the fact that for each $m$ and $n$ the local FW dispersion relation allows two values of $\kappa_{m n}$ (hence of $\delta$ in eqn (34)) with opposite sign. The full-wave solver, on the other hand, does not provide a value for $k_{\perp}$ in the argument of the Bessel functions: this value has to be obtained by separately solving the local FW dispersion relation. Thus the validity of an Eikonal ansatz in the radial coordinate near IC resonances, as in eqn (29), has nevertheless to be assumed. 
In conclusion, using the standard quasilinear procedure, we have derived from Vlasov equation a bounceaveraged QL operator which includes the description of rf-induced radial diffusion, and is equivalent to the QL operator obtained in action-angle variables with the formalism introduced by Kaufman [11]. By assuming the wave field to be known as a superposition of Fourier modes in the toroidal and poloidal angles, we have put the QLDC in a form which can be naturally evaluated using the output of a spectral solver of the wave equations in the plasma. This has actually more than a practical significance. As mentioned in section 2, it can be easily shown that the expression (26) for the quasilinear heating rate is identical with the wave absorption rate predicted by the wave equations derived in [14] by integrating the linearized VLasov equation. This proves that the combination of a constitutive relation derived from the linear Vlasov equation for the wave propagation and absorption with the quasilinear kinetic equation for the slow evolution of the background distribution functions is a complete and internally consistent model for the description of heating and current drive in toroidal plasmas, just as in the infinite homogeneous limit $[20]$.

\section{References}

[1] R.D. Hazeltine, S.M. Mahajan, and D.A. Hitchcock, Phys. Fluids 24 (1981) 1164.

[2] S. Riyopulos, T. Tajima, T. Hatori, and D. Pfirsch, Nucl. Fus. 26 (1986) 627.

[ 3] T. Hellsten, Plasma Phys. Contr. Fus. 31 (1989) 1391.

[4] M.J. Mantsinen, L.-G. Eriksson, C. Gormezano, N.C Hawkes, et al., Nucl. Fus. 40 (2000) 1773.

[ 5] L.-G. Eriksson and P. Helander, Phys. Plasmas 1 (1994) 308.

[ 6] L.-G. Eriksson, M.J. Mantsinen, T Hellsten, and J. Carlsson, Phys. Plasmas 6 (1999) 513.

[ 7] L.-G. Eriksson and M. Schneider, Phys. Plasmas 12 (2005) 072524.

[ 8] J. Carlsson, L.-G. Eriksson and T. Hellsten, Nucl. Fus. 37 (1997) 719.

[ 9] M.J. Mantsinen, L.-G. Eriksson, C. Gormezano, N.C Hawkes, et al., Nucl. Fus. 40 (2000) 1773.

[10] T. Johnson, T. Hellsten, and L.-G. Eriksson, Nucl. Fus. 46 (2006) S433.

[11] A.N. Kaufman, Phys. Fluids 15 (1972) 1063.

[12] I.B. Bernstein and D.C. Baxter, Phys. Fluids 24 (1981) 108.

[13] M. Brambilla, Plasma Phys. Contr. Fus. 41 (1999) 1.

[14] M. Brambilla, Plasma Phys. Contr. Fus. 41 (1999) 775.

[15] I.B. Bernstein, and K. Molvig, Phys. Fluids 26 (1983) 1488.

[16] C.S. Chang, Phys. Fluids 28 (1985) 3598.

[17] L. Chen, L. Vaclavik, and G.W. Hammett, Nucl. Fus. 28 (1988) 389.

[18] P.J. Catto and J.R. Myra, Phys. Fluids B 4 (1992) 187.

[19] P.J. Catto, J.R. Myra, and D.A. Russel, Phys. Plasmas 1 (1994) 52.

[20] M. Brambilla, Kinetic theory of plasma waves, Clarendon Press, Oxford 1998, section 38. 